\title{
Novel Mutation Sites in the Development of Vancomycin- Intermediate Resistance in Staphylococcus aureus
}

\author{
Yubing Wang ${ }^{1}$, Xiaoli $\mathrm{Li}^{1}$, Libo Jiang ${ }^{1,2}$, Wentao Han ${ }^{1}$, Xiangming Xie ${ }^{1}$, Yi Jin ${ }^{1,2 *}$, \\ Xiaoqing $\mathrm{He}^{1,2 *}$ and Rongling $\mathrm{Wu}^{1,2,3 *}$
}

${ }^{1}$ College of Biological Sciences and Biotechnology, Beijing Forestry University, Beijing, China, ${ }^{2}$ Center for Computational Biology, Beijing Forestry University, Beijing, China, ${ }^{3}$ Center for Statistical Genetics, Pennsylvania State University, Hershey, $P A, U S A$

Increased use of vancomycin has led to the emergence of vancomycin-intermediate

OPEN ACCESS

Edited by:

Octavio Luiz Franco

Universidade Católica de Brasilia

(UCB), Brazil

Reviewed by:

Sérgio Alencar,

Universidade Católica de Brasilia

(UCB), Brazil

Carina Elisei Oliveira,

Universidade Católica Dom Bosco

(UCDB), Brazil

*Correspondence:

Yi Jin

wizard.jin@163.com

Xiaoqing $\mathrm{He}$

hexiaoqing.china@126.com

Rongling Wu

rwu@bjfu.edu.cn;

rwu@phs.psu.edu

Specialty section:

This article was submitted to Antimicrobials, Resistance and

Chemotherapy

a section of the journal

Frontiers in Microbiology

Received: 02 September 2016 Accepted: 22 December 2016

Published: 10 January 2017

Citation:

Wang Y, Li X, Jiang L, Han W, Xie X, Jin Y, He X and WU R (2017) Novel Mutation Sites in the Development of Vancomycin- Intermediate Resistance

in Staphylococcus aureus.

Front. Microbiol. 7:2163. doi: 10.3389/fmicb.2016.02163
Staphylococcus aureus (VISA). To investigate the mechanism of VISA development, 39 methicillin-susceptible strains and 3 MRSA strains were treated with vancomycin to induce non-susceptibility, and mutations in six genes were analyzed. All the strains were treated with vancomycin in vitro for 60 days. MICs were determined by the agar dilution and E-test methods. Vancomycin was then removed to assess the stability of VISA strains and mutations. Following 60 days of vancomycin treatment in vitro, 29/42 VISA strains were generated. The complete sequences of rpoB, vraS, graR, graS, walK, and walR were compared with those in the parental strains. Seven missense mutations including four novel mutations (L466S in rpoB, R232K in graS, I594M in walk, and A111T in walR) were detected frequently in strains with vancomycin $\mathrm{MIC} \geq 12 \mu \mathrm{g} / \mathrm{mL}$. Jonckheere-Terpstra trend test indicated these mutations might play an important role during VISA evolution. After the vancomycin treatment, strains were passaged to vancomycin-free medium for another 60 days, and the MICs of all strains decreased. Our results suggest that rpoB, graS, walk, and walR are more important than vraS and graR in VISA development.

Keywords: Staphylococcus aureus, vancomycin, drug-resistance, mutations, jonckheere-terpstra trend test

\section{INTRODUCTION}

Multiple antibiotic resistant Staphylococcus aureus continues to be one of the most common pathogens of both hospital-associated and community-associated infections worldwide (Klevens et al., 2007; Popovich et al., 2007; Hidron et al., 2008; Kallen et al., 2010). Methicillin-resistant S. aureus (MRSA) infection, acquired immunodeficiency syndrome (AIDS) and viral hepatitis $\mathrm{B}$ are the three major infectious diseases worldwide and pose a serious threat to public health (Dantes et al., 2013). Vancomycin is the first-line antibiotic therapy for MRSA infections (Sieradzki et al., 1999; Deresinski, 2005; Moellering, 2005). However, increased use of vancomycin has led to the emergence of vancomycin-intermediate S. aureus (VISA) (Hiramatsu et al., 1997b). Currently, the Clinical Laboratory Standards Institute (CLSI) categorizes $S$. aureus as vancomycin susceptible (VSSA) (MIC $\leq 2$ $\mu \mathrm{g} / \mathrm{mL}$ ), vancomycin intermediate resistant $(4-8 \mu \mathrm{g} / \mathrm{mL}$ ), and vancomycin resistant (VRSA) $(\mathrm{MIC} \geq 16 \mu \mathrm{g} / \mathrm{mL}$ ) (Patel, 2014). VISA has been reported more frequently worldwide and 
has aroused considerable concern (Hiramatsu et al., 1997a, 2002; Tenover and Moellering, 2007; Rishishwar et al., 2016).

VRSA emerged in 1997 due to acquisition of the vanA gene from vancomycin-resistant enterococci (Hiramatsu et al., 1997a; Chang et al., 2003). Previous studies indicated that spontaneous mutations play important roles in the evolution of drug resistance (Drlica, 2003; Andersson and Hughes, 2014). However, the genetic mechanism of vancomycin resistance in VISA strains has not been identified fully (Cameron et al., 2016). Several of mutations in multiple genes and gene regulation systems are needed to achieve a VISA phenotype. Several genetic alterations in two-component regulatory systems have been reported to be strongly associated with a VISA phenotype, including mutations in the vraSR operon (Mwangi et al., 2007), graRS (Howden et al., 2008b; Neoh et al., 2008; Cui et al., 2009), and walRK (Howden et al., 2011; Shoji et al., 2011). VraS may serve as a switch for the activity of the "cell wall stimulon" (Gardete et al., 2012). The vraS I5N mutation was found to confer heterogeneous vancomycin resistance when introduced into a vancomycin-susceptible MRSA strain (Katayama et al., 2009). The graR N197S mutation was suggested to convert strain Mu3 into the VISA phenotype (Neoh et al., 2008). Howden et al. confirmed that the T136I mutation in graS is also a key mediator of vancomycin resistance (Howden et al., 2008b). The G223D mutation in walK and the K208R mutation in walR were associated with increased vancomycin MICs (Howden et al., 2011; Shoji et al., 2011). The rpoB gene, which encodes an RNA polymerase subunit, also plays an important role in the evolution of VISA (Matsuo et al., 2011). The H481Y mutation in $r p o B$ has been confirmed by allelic replacement experiments to increase vancomycin resistance during development of the VISA phenotype in the Mu3 (hVISA) strain (Matsuo et al., 2011). The majority of previous reports on the genetic mechanism of VISA development have focused on clinical MRSA strains (Doddangoudar et al., 2012). The development of vancomycin non-susceptibility might be affected by methicillin or other drug resistance, and so VISA development in methicillin-susceptible strains warrants investigation.

In this study, 39 methicillin-susceptible $S$. aureus and 3 MRSA strains were treated with increasing concentrations of vancomycin in vitro to investigate the genetic mechanism underlying development of vancomycin resistance. The genes (rpoB, vraS, graSR, and walRK) important for development of vancomycin non-susceptibility were analyzed after 60 days of vancomycin treatment and compared with those in the parental strains. Our results suggest that four novel mutation sites are important for VISA development: L466S in rpoB, R232K in graS, I594M in walK, and A111T in walR.

\section{MATERIALS AND METHODS}

\section{Bacterial Strains}

Forty-two VSSA strains (numbered as S1-S42) used in this study were purchased from the China General Microbiological Culture Collection Center, China Center of Industrial Culture Collection, Agricultural Culture Collection of China, China Forestry Culture Collection Center, China Center for Type Culture Collection, China Pharmaceutical Culture Collection, National Center for
Medical Culture Collections, and China Agricultural University. All strain's background were described in Table 1. Only S22 and S24 were obtained from patients, others were separated from animals or environment. Among these 42 strains, three were MRSA (S3, S34, and S37). All strains were stored at $-80^{\circ} \mathrm{C}$.

\section{Antimicrobial Susceptibility Testing}

The vancomycin MICs of all $S$. aureus parental strains were determined by standardized agar dilution methods, according to the CLSI guidelines (Cockerill, 2012). E-tests were performed using glycopeptide resistance detection strips (bioMérieux), including vancomycin, oxacillin, rifampicin, teicoplanin, according to the manufacturer's instructions. For determination of MICs, a single colony was inoculated in brain-heart infusion (BHI) broth (Oxoid, Basingstoke, UK) and incubated at $37^{\circ} \mathrm{C}$. At a cell density of $0.5 \mathrm{McFarland}$ units $\left(10^{8} \mathrm{CFU} / \mathrm{mL}\right)$, bacteria were streaked evenly onto MuellerHinton agar (Oxoid, Basingstoke, UK) plates. Plates were incubated at $37^{\circ} \mathrm{C}$, and the MICs were read after $18-24 \mathrm{~h}$ of incubation.

\section{In vitro Development of Vancomycin Non-susceptibility}

All S. aureus strains were incubated on BHI agar (Oxoid, Basingstoke, UK) plates with vancomycin (Sigma-Aldrich, St. Louis, MO, USA) at $50 \%$ of the initial MIC. Plates were incubated at $37^{\circ} \mathrm{C}$, and the strains were passaged to fresh medium containing the same vancomycin concentration every $24 \mathrm{~h}$. MICs were re-determined after 4 days of treatment using the $E$-test method. The vancomycin concentration was increased to $50 \%$ of the new MIC level of each strain. This process was repeated every 4 days for 60 days. Stability of VISA strains was then determined by passaging onto vancomycin-free agar plates every $24 \mathrm{~h}$ for 60 days.

\section{Sequence Analysis and Mutation Detection}

To identify the point mutations and amino acid changes between 60-day-treated and parental strains, the rpoB, vraS, graS, graR, $w a l K$, and walR genes of all treated and parental strains were amplified using the primers shown in Table 2. One colony of each strain was treated with lysostaphin and lysozyme, and genomic DNA was extracted using a TIANamp bacteria DNA kit (Tiangen, China) according to the manufacturer's instructions. PCR amplification using genomic DNA as the template was performed using Ex Taq DNA polymerase (Takara Shuzo Co., Ltd, Kyoto, Japan). The amplification conditions for $r p o B$, graS, and walK were as follows: $94^{\circ} \mathrm{C}$ for $3 \mathrm{~min}, 30$ cycles of $94^{\circ} \mathrm{C}$ for 1 $\min , 55^{\circ} \mathrm{C}$ for $1 \mathrm{~min}$, and $72^{\circ} \mathrm{C}$ for $1 \mathrm{~min}$, followed by a final step at $72^{\circ} \mathrm{C}$ for $7 \mathrm{~min}$. The amplification conditions for vraS, graR, and walR were as follows: $94^{\circ} \mathrm{C}$ for $3 \mathrm{~min}, 30$ cycles of $94^{\circ} \mathrm{C}$ for $1 \mathrm{~min}, 57^{\circ} \mathrm{C}$ for $1 \mathrm{~min}$, and $72^{\circ} \mathrm{C}$ for $1 \mathrm{~min}$, followed by a final step at $72^{\circ} \mathrm{C}$ for $7 \mathrm{~min}$. PCR products were purified, and their sequences were analyzed. Nucleotide and amino acid sequence comparisons of each pair of treated and parental strains were performed using DNAMAN8.0 (Lynnon Biosoft, USA). Based on the amino acid sequence alignment, the missense mutations were analyzed by Jonckheere-Terpstra trend test. 
TABLE 1 | All strains' background.

\begin{tabular}{|c|c|c|c|c|c|}
\hline & Strain ID & Provider & & Strain ID & Provider \\
\hline S2 & $21600\left(\right.$ ATCC $\left.27217^{C}\right)$ & $\mathrm{ClCC}$ & S23 & 22944 & $\mathrm{ClCC}$ \\
\hline S4 & 01340 & ACCC & S25 & AB 91119 & ССТСС \\
\hline S5 & 01332 & ACCC & S26 & 10201 & $\mathrm{ClCC}$ \\
\hline S8 & 10341 & CFCC & S29 & 141405 & CPCC \\
\hline S9 & 1.8721 (ATCC 29213) & CGMCC & S30 & 26003 & $\mathrm{CMCC}$ \\
\hline S10 & 1.1697 & CGMCC & S31 & 26112 & CMCC \\
\hline S11 & 1.1476 & CGMCC & S32 & 01011 & ACCC \\
\hline S12 & 141396 & CPCC & S33 & 26001 & CMCC \\
\hline S17 & 22942 & $\mathrm{ClCC}$ & S38 & AB18 & CAU \\
\hline $\mathrm{S} 18$ & AB 94004 & ССТСС & S39 & CD1 & CAU \\
\hline S19 & AB 91093 & ССТСС & S40 & CD9 & CAU \\
\hline S20 & AB 91053 & сСтсС & S41 & $\mathrm{CD7}$ & CAU \\
\hline S21 & 23699 & $\mathrm{ClCC}$ & S42 & 01336 & ACCC \\
\hline
\end{tabular}

a Strain number; ${ }^{b}$ Center ID; ${ }^{C}$ ATCC ID.

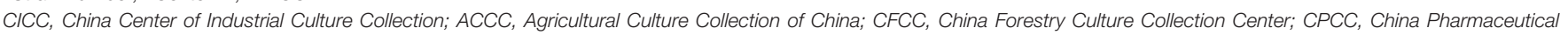

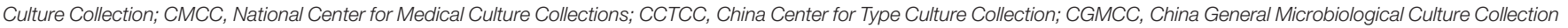
Center; CAU, China Agricultural University.

TABLE 2 | Sequences of Primers.

\begin{tabular}{llc}
\hline Gene & $\mathbf{5}^{\prime}$-3' primer sequence & Product length (bp) \\
\hline vras F & GACGTAGAGGTGATTATCGATGAACCACT & 1044 \\
vraS R & TTAATCGTCATACGAATCCTCCTTATTAA & \\
graS F & ATGAGTATGGAACTTGGGCA & 1592 \\
graS R & TCCCAGATCCAGAGGGACC & \\
graR F & GGATTAAAGATTTCAAAGTC & 675 \\
graR R & GAGATTCAAAAATAAGCTAC & \\
walR F & ACCAGGTTGGACAGAAGACG & 2000 \\
walR R & TGTGCATTACGGAGCCCT & \\
walk F & CGCGTAGAGGCGTTGGATA & 1983 \\
walk R & TGGCTGTCATAGGTGTCGTT & \\
rpoB F1 & GCAAGGTATGCCATCTGCAAAG & 1954 \\
rpoB R1 & TTGCTTCGGCGATACATCCA & \\
rpoB F2 & ACGTGAACGTGCTCAAATGG & 2262 \\
rpoB R2 & ATGCCTTGTAGCGAACACG &
\end{tabular}

The presence of mecA and vanA in all strains was detected as described previously using the following primers: $m e c A$ forward 5'-TGGCTATCGTGTCACAATCG-3'; reverse $5^{\prime}$-CT GGAACTTGTTGAGCAGAG-3'; vanA forward 5' -ATGAATAG AATAAAAGTTGC-3'; reverse $5^{\prime}$-TCACCCCTTTAACGCTAA TA-3' (Saha et al., 2008). The multilocus sequence typing (MLST) genotypes of all strains were also determined as described
(Enright et al., 2000). Seven housekeeping genes of all parental strains were sequenced to obtain the sequence type (ST) of each strain.

\section{Statistical Analysis}

Based on the relationships between the MICs and time points during the vancomycin treatment, the 42 strains were grouped by hierarchical clustering (Tibshirani et al., 2001). After the in vitro treatment, genotypes were coded as 0,1 , or 2 for each SNP in sequenced genes. The association between each mutation site and MICs measured after 60 days vancomycin treatment was analyzed using the Jonckheere-Terpstra (JT) trend test (Jonckheere, 1954). $P<0.05$ were considered to indicate significance. All statistical analyses were performed using the $\mathrm{R}$ statistical software (version 2.1).

\section{RESULTS}

\section{Development of Vancomycin-Intermediate Resistance in S. aureus In vitro}

Forty-two VSSA strains were treated with vancomycin in vitro for 60 days. After induction, although the MICs varied, only the MIC of the S10 strain remained unchanged compared with its parental strain. In contrast, the MICs of many strains increased significantly (Figure 1). Twenty-nine VISA strains were generated within 60 days, while 13 strains remained 

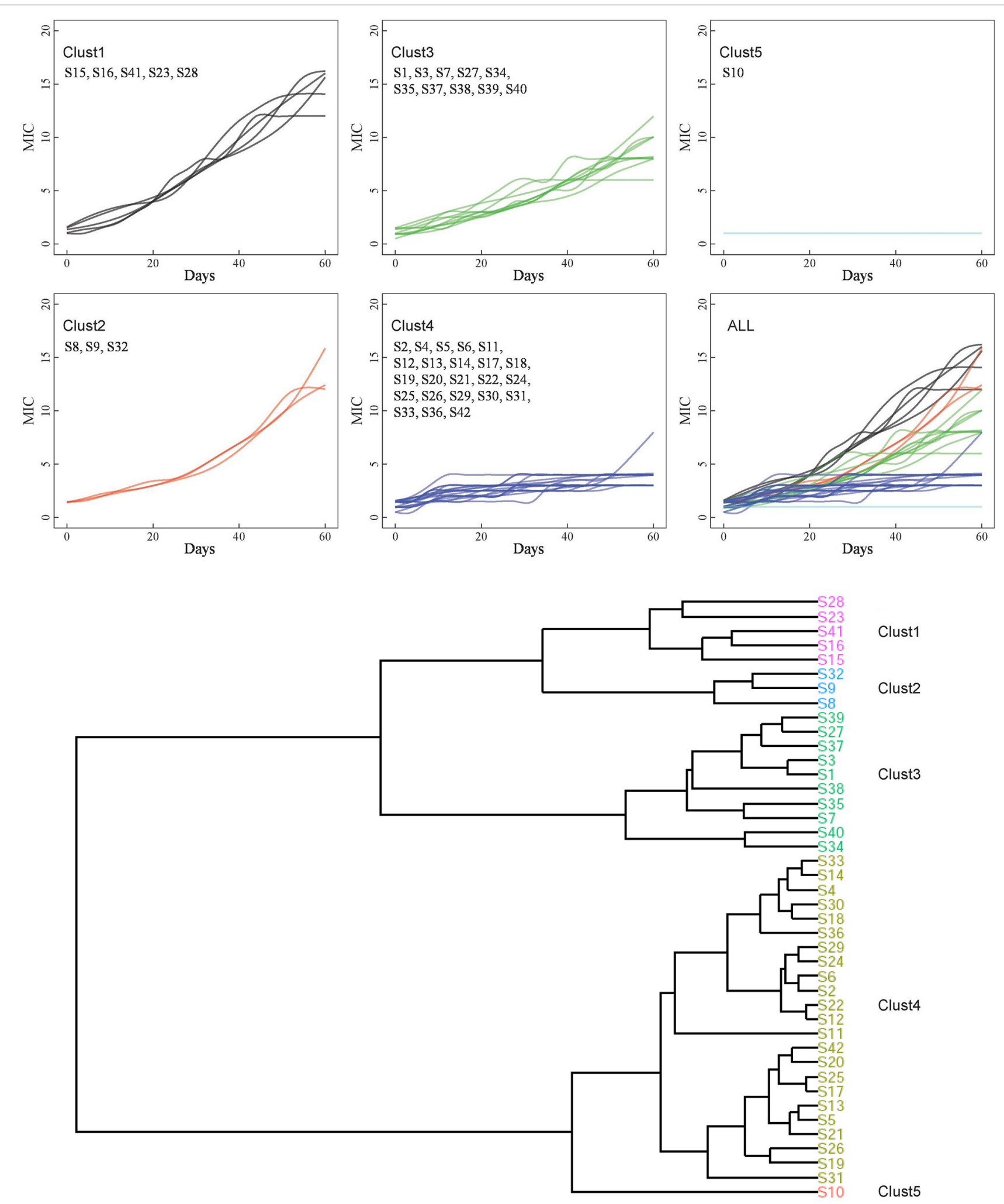

FIGURE 1 | Hierarchical clustering of $\mathbf{4 2}$ strains. Based on the association between MICs and time point, the entire 42 strains were grouped into 5 clusters by hierarchical clustering. 
vancomycin susceptible (MIC $<4 \mu \mathrm{g} / \mathrm{mL}$ ). The maximum MIC of $16 \mu \mathrm{g} / \mathrm{mL}$ was achieved in 4/29 strains: S8, S15, S16, and S41. The MICs of these four strains met the standard for VRSA rather than VISA strains; however, the vanA gene cluster, a common vancomycin-resistance determinant, was absent (data not shown). The MICs of all strains were listed in Table 3.

Based on the relationships between the MICs and time points, the 42 strains were grouped into five clusters by hierarchical clustering (Figure 1). Eight strains were found in $\mathrm{C} 1$ and $\mathrm{C} 2$ and showed the highest MICs $(12-16 \mu \mathrm{g} / \mathrm{mL})$. These strains exhibited rapid development of vancomycinintermediate resistance, which was accompanied by several common mutations in sequenced genes, such as L466S and $\mathrm{H} 481 \mathrm{~N}$ in $r p o B$, R232K in graS, R222K, A468T, and I594M in walK, and A111T in walR (Table 3).

The third cluster comprised 10 strains. The MICs of these strains increased gradually over time, reaching 6-12 $\mu \mathrm{g} / \mathrm{mL}$. Twenty-three strains, including 12 VSSA strains, were grouped in $\mathrm{C} 4$ and exhibited slow development of vancomycin nonsusceptibility, resulting in MICs of 3-8 $\mu \mathrm{g} / \mathrm{mL}$. Only the MIC of $\mathrm{S} 11$, which harbored the A243T mutation in walK, increased to 8 $\mu \mathrm{g} / \mathrm{mL}$ (Table 3). Only strain S10 was grouped in C5. The MIC of S10 was unchanged after the 60 days of treatment.

The sequence type (ST) of all parental strains was shown in Table 3. Most (8/9) of the strains with MICs $\geq 12 \mu \mathrm{g} / \mathrm{mL}$ were assigned to different ST types.

\section{Antibiotic Susceptibilities of All S. aureus Strains}

To determine susceptibilities to other antibiotics after 60 days of treatment, the oxacillin, rifampicin, and teicoplanin MICs of all strains were measured by the E-test. The MICs of several strains from each cluster are shown in Table 4. All of the 42 VSSA parental strains were initially susceptible to teicoplanin, three (S3, S34, and S37) were resistant to oxacillin, and two (S3 and S16) were resistant to rifampicin. After vancomycin treatment, the teicoplanin MICs of most strains increased, while the oxacillin and rifampicin MICs of most strains did not change. In contrast, the oxacillin MICs of five strains (S3, S15, S16, S34, and S37) decreased. Three VISA strains (S1560, S35 60 , and S37 60 ) became resistant to rifampicin, and the five rifampicin resistant strains carried Asn (N) in the 481st amino acid of $r p o B$ (Table 3). However, the parental strains $S 37$ and $S 41_{60}$, which also carried this Asn $(\mathrm{N})$, were susceptible to rifampicin.

\section{Amino Acid Mutations Involved in VISA Development}

The complete sequences of rpoB, vraS, graS, graR, walK, and walR were amplified from all strains. Only missense mutations were observed by amino acid sequence alignment between the 60 days' vancomycin-treated strains and parental strains. A large number of SNPs were detected, and the mutations varied among the strains. Multiple nonsynonymous mutations in the six genes are shown in Table 3.

In $r p o B$, we found 10 distinct amino acid changes in 13/29 (44.8\%) VISA strains: F279L, L466S, H481N, T518M, D631E, Y737F, R917S, D1046V, and T1182I/I1182T. No amino acid substitution was found at the 466th or 481st locus in the
VSSA strains treated by vancomycin for 60 days. Notably, the T1182I/I1182T mutations not only occurred frequently in VISA strains but were also found in VSSA strains. In vraS, seven distinct missense mutations were identified in 10/29 (34.5\%) VISA strains. No nonsynonymous SNPs were found in the other VISA or VSSA strains. In the graRS operon, 19 distinct mutations were identified in 20/29 (69.0\%) VISA strains. The L26F/F26L, I59L/L59I, and Y223D mutations were present in both VISA and VSSA strains, while the D223Y and T224I mutations were detected only in VSSA strains. Twenty-two of the 29 (75.9\%) VISA strains harbored mutations in the walRK operon, including 23 distinct mutations in walK and 5 in walR. Among these mutations, R222K, A468T, and I594M in walK and A111T in walR occurred more frequently. Furthermore, all VSSA strains lacked R222K, I594M, and A111T but carried several other mutations.

Overall, three VISA strains (S15, S35, and S41) contained the L466S and $\mathrm{H} 481 \mathrm{~N}$ mutations in $r p o B$, accompanied by the mutations $\mathrm{R} 232 \mathrm{~K}$ in graS, R222K, A468T, and I594M in walK, and A111T in walR and acquired high-level vancomycin resistance $(\mathrm{MIC} \geq 12 \mu \mathrm{g} / \mathrm{mL}$ ). The walK mutation was carried most frequently by VISA strains. A previous report stated that mutations in walK were most frequent in 39 clinical VISA strains from various countries (Shoji et al., 2011).

To analyze the associations between mutation sites and MICs, strain genotypes were coded as 0,1 , or 2 for each mutation in sequenced genes. The associations between mutations and MICs at 60 days were analyzed using the Jonckheere-Terpstra (JT) trend test. As shown in Figure 2, 10 mutations (L466S and $\mathrm{H} 481 \mathrm{~N}$ in $r p o B, \mathrm{~V} 15 \mathrm{G}$ in $v r a S, \mathrm{~L} 26 \mathrm{~F}, \mathrm{I} 5 \mathrm{~L}, \mathrm{Y} 223 \mathrm{D}$, and $\mathrm{R} 232 \mathrm{~K}$ in graS, R222K, and I594M in walK and A111T in walR) were significantly correlated with the MIC differences, including four novel mutation sites (L466S in rpoB, R232K in graS, I594M in walK and A111T in walR). These four mutation sites were only occurred in five strains with MICs $\geq 10 \mu \mathrm{g} / \mathrm{mL}$ and have not been reported to date. The $P$ values of all mutations in graR were $>0.05$, indicating that this gene might be relatively unimportant in the process of VISA development.

\section{Stability of Vancomycin Non-susceptibility}

To investigate the stability of vancomycin non-susceptibility, strains were incubated on vancomycin-free agar plates for another 60 days after the vancomycin treatment. The MICs of all strains (except S10) decreased after the removal of vancomycin, while S35 and S41 remained as VISA strains. Six genes $(r p o B$, vraS, graS, graR, walK, and walR) were sequenced and compared with the genes before vancomycin removal. The strains with mutations were shown in Table 5; 27 reverse mutations and 13 random mutations were detected compared with those vancomycin treated strains. Only the reverse mutation F10L in walK might be related to a decrease in the MIC.

\section{DISCUSSION}

VISA strains are increasingly prevalent in the hospital setting and are a major issue in the treatment of MRSA infections. It has been suggested that the occurrence of VISA strains is relatively frequent, representing a threat to public health (Sader 
TABLE 3 | Amino acids mutations in VISA development.

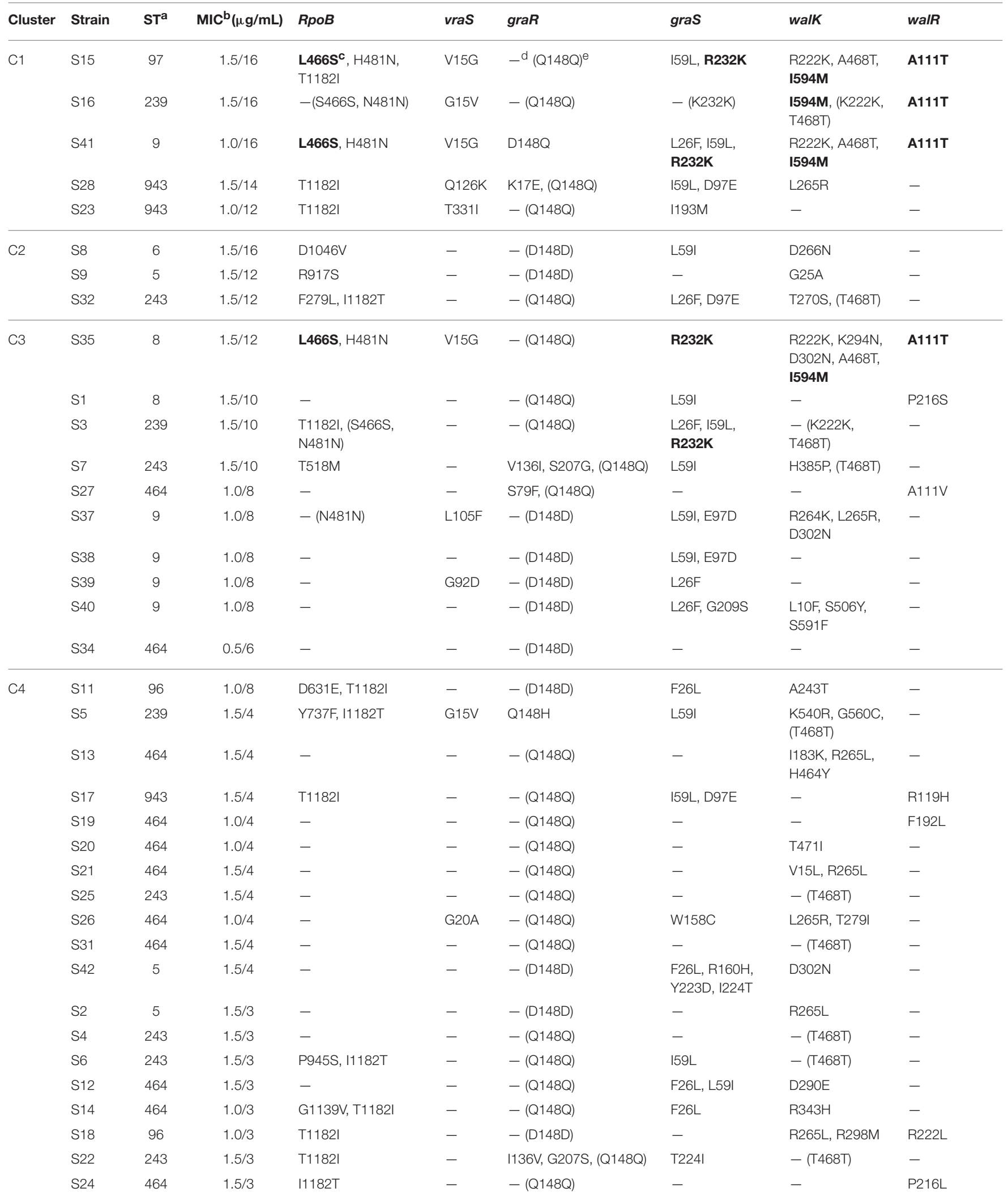


TABLE 3 | Continued

\begin{tabular}{|c|c|c|c|c|c|c|c|c|c|}
\hline Cluster & Strain & $\mathbf{S T} \mathbf{T}^{\mathrm{a}}$ & $\operatorname{MIC}^{b}(\mu \mathrm{g} / \mathrm{mL})$ & RpoB & vras & graR & gras & walk & walR \\
\hline & S30 & 464 & $0.5 / 3$ & - & - & - (Q148Q) & - & - & $\mathrm{R} 107 \mathrm{H}$ \\
\hline & S36 & 770 & $0.5 / 3$ & - & - & - (D148D) & L26F, D223Y & - & - \\
\hline
\end{tabular}

aST, sequence type.

${ }^{b}$ MIC $(\mu \mathrm{g} / \mathrm{mL})$, MICs of parental/vancomycin treated strains.

${ }^{c}$ Bold indicates the novel mutations.

${ }^{d}$ Dash indicates amino acids had no change after 60 days vancomycin treatment.

e Parentheses indicates the amino acids remain unchanged.

TABLE 4 | Antibiotic susceptibilities changes of strains.

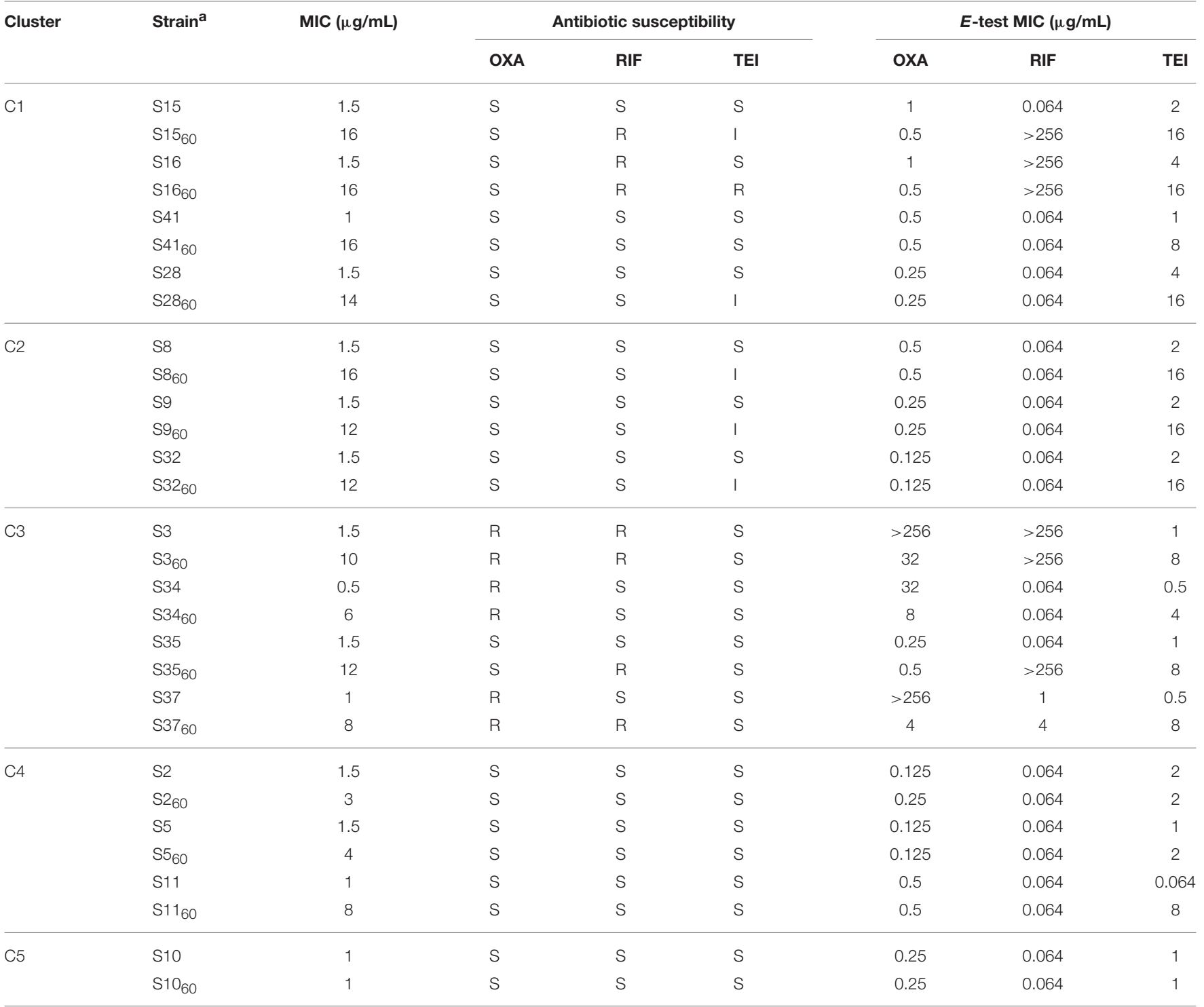

OXA, oxacillin; RIF, rifampicin; TEl, teicoplanin. S, susceptible; I, intermediate; $R$, resistant.

${ }^{a}$ No subscript represents parental strains.

Subscript indicates strains treated by vancomycin for 60 days. 


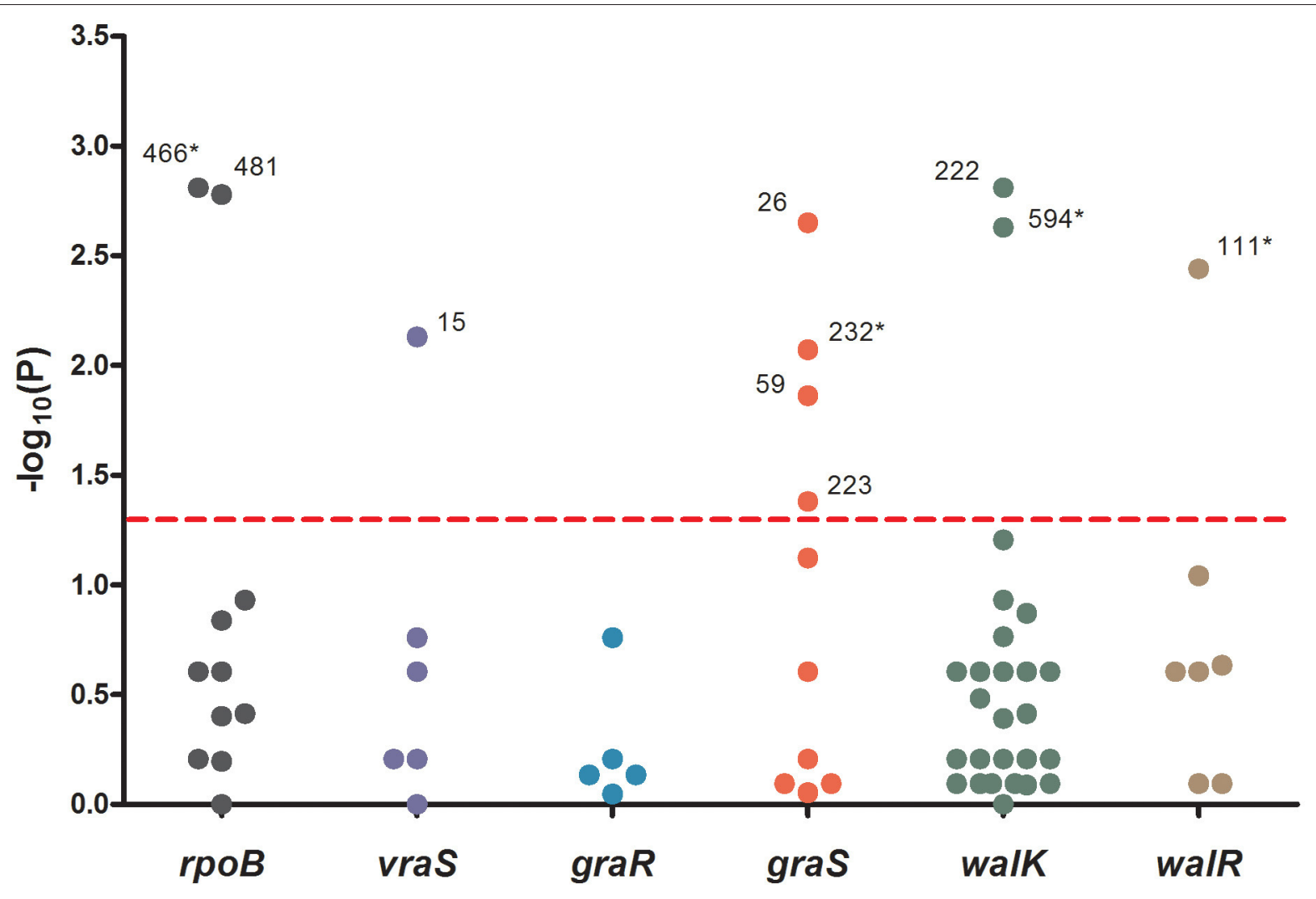

FIGURE 2 | The significance of association between each mutation sites and MICs. Data was analyzed by Jonckheere-Terpstra (JT) trend test. The $x$ axis shown six sequenced genes and the y axis shown - log10 of the $P$ values resulting from the JT trend test. Each dot represented a mutation site, and the number beside the dot shown the amino acid position. $P<0.05$ were considered significant. ${ }^{*}$ The novel mutation sites found in this study.

et al., 2009). Prolonged vancomycin exposure in patients can contribute to generation of VISA, resulting in treatment failure (Liu et al., 2011). In addition, VISA can also be generated in vitro from VSSA strains by exposure to vancomycin (Matsuo et al., 2011; Doddangoudar et al., 2012). Previous studies of the mechanisms of VISA development focused on clinical MRSA strains (Doddangoudar et al., 2012), while few studies have addressed VISA development in methicillin-susceptible strains. In this study, 29 (69\%) VISA strains were generated from 42 S. aureus strains by vancomycin treatment for 60 days in vitro. Our results suggest that seven mutation sites are important for VISA development, including four novel mutations: L466S in $r p o B$, $\mathrm{R} 232 \mathrm{~K}$ in graS, I594M in walK, and A111T in walR.

Hierarchical clustering which can directly decompose the dataset into a set of disjoint clusters was performed to trace the dynamic changes in VISA development, and investigate different vancomycin non-susceptibility evolution patterns. The 42 strains were grouped into five clusters, and the MICs of nine strains (eight from C1 and C2 and one from C3) were $\geq 12 \mu \mathrm{g} / \mathrm{mL}$ (Figure 1). Among those nine strains, only S28 and S23 were assigned to the same ST type (Table 3). 38/42 parental strains was susceptible to oxacillin, rifampicin and teicoplanin. The three MRSA strains (S3, S34, and S37) were resistant to oxacillin, and 2/42 strains (S3 and S16) were resistant to rifampicin.
Strains S3 and S16 developed high MICs $(10-16 \mu \mathrm{g} / \mathrm{mL})$ after vancomycin treatment. Three methicillin-susceptible strainsS8, S15, and S41 - showed the highest MIC of $16 \mu \mathrm{g} / \mathrm{mL}$. There was no significant correlation between VISA development and initial resistance to oxacillin, rifampicin or teicoplanin. The oxacillin MICs of five strains (S3, S15, S16, S34, and S37) decreased after vancomycin treatment. It has been suggested that upon acquisition of vancomycin resistance, some strains show a concomitant decrease in oxacillin resistance (Bhateja et al., 2006), and a previous study reported that mutated graR may impair oxacillin resistance (Neoh et al., 2008). In this study, no graR mutation was detected in these five strains. The H481Y/N mutation is located in the rifampin resistancedetermining region, and this locus has been reported repeatedly in clinical rifampicin-resistant $S$. aureus strains (Aubry-Damon et al., 1998; O’Neill et al., 2006; Mick et al., 2010). In this study, five rifampicin-resistant strains harbored the Asn $(\mathrm{N})$ mutation in the 481st amino acid of $r p o B$ (Table 3). However, the parental strains S37 and $\mathrm{S} 41_{60}$, which harbor Asn (N), were susceptible to rifampicin. In summary, no significant association was found between these mutations and antibiotic susceptibility changes.

The genetic basis for vancomycin resistance in VISA remains unclear. The rpoB, vraSR, graSR, and walRK genes have been reported to be highly associated with vancomycin resistance. In 
TABLE 5 | Mutations after removal of vancomycin.

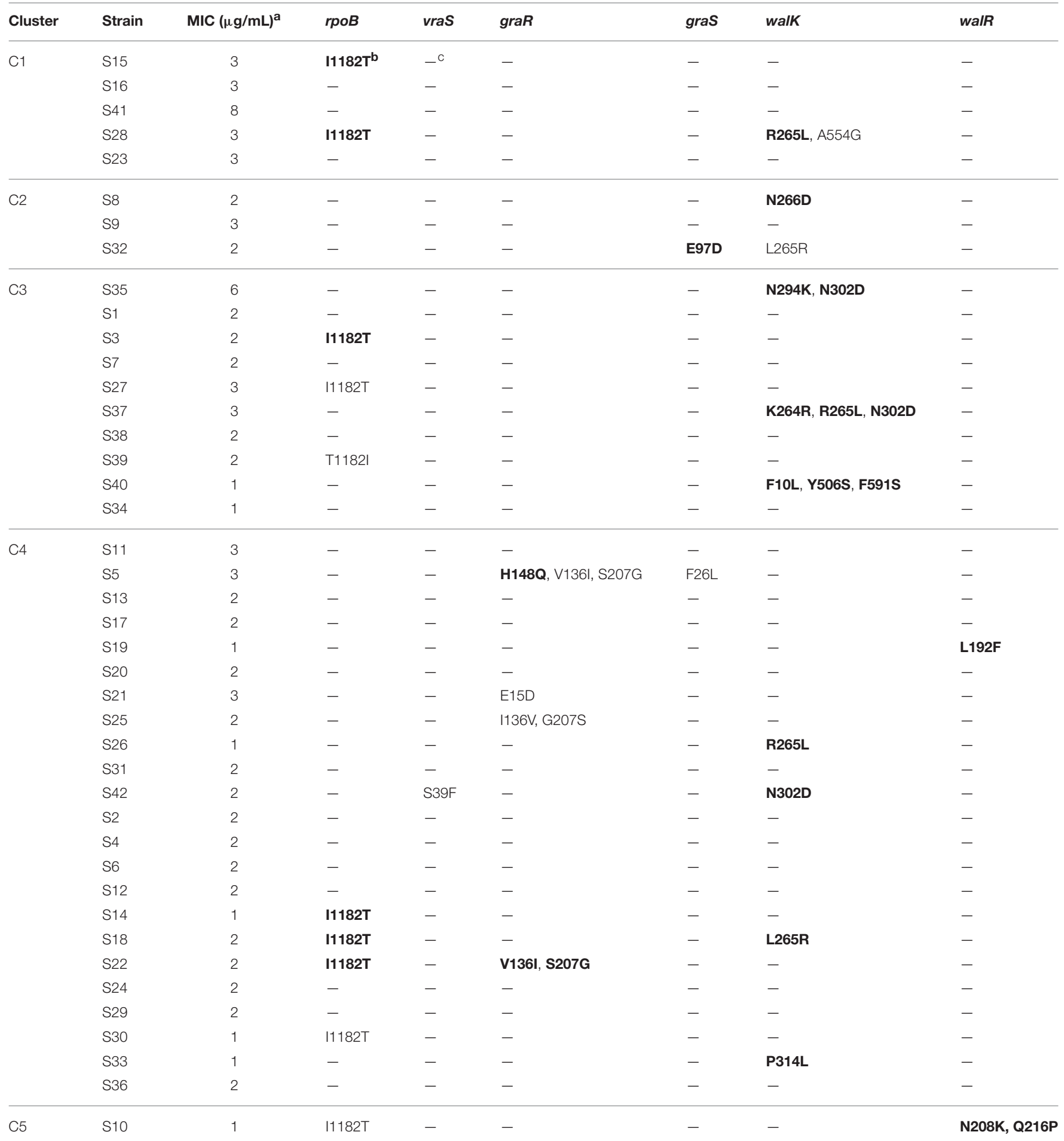

${ }^{a}$ MIC $(\mu \mathrm{g} / \mathrm{mL})$, MICs after vancomycin removal for 60 days.

${ }^{b}$ Bold indicates reverse mutations.

${ }^{c}$ Dash indicates the amino acids remain unchanged.

this study, the complete sequences of rpoB, vraS, graS, graR, walK, and walR were analyzed and compared with those of the susceptible parental strains. Seven mutations occurred more frequently in strains with high MICs $(12-16 \mu \mathrm{g} / \mathrm{mL})$, including L466S and $\mathrm{H} 481 \mathrm{~N}$ in $r p o B, \mathrm{R} 232 \mathrm{~K}$ in gras, $\mathrm{R} 222 \mathrm{~K}, \mathrm{~A} 468 \mathrm{~T}$, and I594M in walK, and A111T in walR (Table 3). In vitro experiment 
and JT trend test indicated that four novel mutation sites were important for VISA development-L466S, R232K, I594M, and A111T-which were first reported in this study (Figure 2).

Alam et al. reported that $r p o B \mathrm{H} 481$ is the predominant locus associated with an increased vancomycin MIC (Alam et al., 2014). The mutations $\mathrm{H} 481 \mathrm{Y} / \mathrm{N}$ in $r p o B$ play a dual role in rifampin and vancomycin resistance (Watanabe et al., 2011; Gao et al., 2013). Moreover, it has been suggested that the $r p o B$ mutation itself, and not any other incidental genetic change caused by rifampin resistance, was responsible for the decreased vancomycin susceptibility (Matsuo et al., 2011). In this study, two mutations (L466S and H481N) were significantly associated with increased vancomycin MICs. Although the L466S mutation had not been reported previously, it might be important for the development of VISA.

In $v \mathrm{raS}$, the 15th amino acid locus was significantly associated with MIC according to the JT trend test, but both the V15G and G15V mutations were detected in different VISA strains. Therefore, V15G/G15V seemed to be less important in VISA development.

D148Q mutation in graR was reported previously to be important for development of high-level resistance (Neoh et al., 2008; Doddangoudar et al., 2012). In this study, only the S41 strain contained the D148Q mutation in $\operatorname{graR}$ (MIC $=16$ $\mu \mathrm{g} / \mathrm{mL}$ ), while $28 / 42(66.7 \%)$ VSSA parental strains initially harbored Gln (Q) at locus 148. Therefore, we speculated that the D148Q mutation in graR did not significantly affect the development of vancomycin resistance. The S79F mutation is important in the development of VISA strains (Neoh et al., 2008; Shoji et al., 2011; Doddangoudar et al., 2012; Hafer et al., 2012). Among our strains, only S27 carried the S79F mutation, and exhibited a MIC of $8 \mu \mathrm{g} / \mathrm{mL}$. According to the JT trend test, no mutation in graR and four mutations (L26F, I59L, $\mathrm{Y} 223 \mathrm{D}$, and $\mathrm{R} 232 \mathrm{~K}$ ) in graS were significantly related to elevated vancomycin MICs. However, the L26F/F26L, I59L/L59I, and Y223D mutations were detected in both VISA and VSSA strains. Thus, these mutation sites were unlikely to be responsible for VISA development. The R $232 \mathrm{~K}$ mutation occurred frequently (3/9) in strains with high MICs $(12-16 \mu \mathrm{g} / \mathrm{mL})$ and was absent in VSSA strains. This suggests that the $\mathrm{R} 232 \mathrm{~K}$ mutation is involved in the development of vancomycin-intermediate resistance.

G223D mutation in walK and K208R mutation in walR were associated with increased vancomycin MICs (Howden et al., 2011; Shoji et al., 2011). However, these genetic changes are not observed frequently in clinical and laboratory $S$. aureus strains (Howden et al., 2008a,b; Kato et al., 2010). Shoji et al. collected 39 clinical VISA strains from various countries worldwide and then analyzed the complete sequences of vraSR, graSR, $c l p P$, and walRK. Nine of the 39 (23\%) VISA strains from four countries harbored R222K and A468T mutations in walK. The L10F and A243T mutations in walK and P216S in walR have been reported previously in VISA strains (Shoji et al., 2011; Hafer et al., 2012). In this study, 3/9 VISA strains with MICs $\geq 12 \mu \mathrm{g} / \mathrm{mL}$ harbored the R222K mutation, S3 and S16 initially carried Lys (K) at locus 222. The A468T mutation occurred in the same three VISA strains as R222K, while other ten strains carried Thr (T) including seven VISA strains initially (Table 3). Therefore, A468T seemed to be important for VISA development. According to the JT trend test, $\mathrm{R} 222 \mathrm{~K}, \mathrm{I} 594 \mathrm{M}$ in walK and A111T in walR were significantly associated with vancomycin resistance. Although the mutations I594M in walK and A111T in walR have not been reported to date, they were frequently detected (4/9) in strains with MICs $\geq$ $12 \mu \mathrm{g} / \mathrm{mL}$. The presence of I594M and A111T in the same strain may result in development of the VISA phenotype.

Overall, seven amino acid changes-L466S, H481N, R232K, R222K, A468T, I594M, and A111T-were detected frequently in our VISA strains. Although the four novel non-synonymous mutations L466S, R232K, I594M, and A111T have not been reported to date, we speculate that these mutations may play an important role in development of VISA strains, together with the H481N, R222K, and A468T mutations, synergistically promote and maintain high-level vancomycin resistance. Several other mutations were detected in both VISA and VSSA strains or in only VSSA strains. Whether these mutations affect development of a VISA phenotype remains unknown. Notably, certain VISA strains did not harbor any important mutations within the sequenced genes. The increased MICs of these strains may be caused by mutations in other undetected/unidentified genes, such as the proteolytic regulatory gene $c l p$ (Shoji et al., 2011) and the accessory gene regulator agr (Sakoulas et al., 2002, 2005).

After removal of vancomycin for 60 days, the MICs of all strains decreased. The L10F mutation in walK has been reported previously in VISA strains, thus, the reverse mutation F10L in walK might be associated with loss of vancomycin resistance. These findings suggest that VISA development is affected by a complex gene regulatory network, although other genes or pathways might be involved in decreased MICs observed. The presence of stop codons in vraS and graR was reported to be related to loss of vancomycin non-susceptibility (Doddangoudar et al., 2012). However, no stop codon was detected in this study.

In conclusion, this study demonstrated that prolonged vancomycin exposure leads to development of vancomycinintermediate resistance in methicillin-susceptible $S$. aureus strains. Compared with the susceptible parental strains, four novel missense mutations-L466S in $r p o B$, R232K in graS, I594M in walK and A111T in walR-were detected in high-level vancomycin-resistant strains. Our results also suggest that $r p o B$, graS, walK and walR are more important than $v \mathrm{raS}$ and graR in the evolution of vancomycin non-susceptibility.

\section{AUTHOR CONTRIBUTIONS}

YJ, XH, and RW conceived and designed the experiments. YW, $\mathrm{XL}, \mathrm{LJ}$, and $\mathrm{WH}$. performed the experiments. XX, YJ, XH, and RW analyzed the data, YW and YJ. wrote the manuscript. All authors reviewed the manuscript.

\section{FUNDING}

This work was supported by the Fundamental Research Funds for the Central University (TD2012-03), the Special Fund for Forest Scientific Research in the Public Welfare (201404102), Natural Science Foundation of China (51108029) and a "One-Thousand Person Plan" award. 


\section{REFERENCES}

Alam, M. T., Petit, R. A., Crispell, E. K., Thornton, T. A., Conneely, K. N., Jiang, Y., et al. (2014). Dissecting vancomycin-intermediate resistance in Staphylococcus aureus using genome-wide association. Genome Biol. Evol. 6, 1174-1185. doi: 10.1093/gbe/evu092

Andersson, D. I., Hughes, D. (2014). Microbiological effects of sublethal levels of antibiotics. Nat. Rev. Microbiol. 12, 465-478. doi: 10.1038/nrmicr o3270

Aubry-Damon, H., Soussy, C. J., and Courvalin, P. (1998). Characterization of mutations in the rpoB gene that confer rifampin resistance in Staphylococcus aureus. Antimicrob. Agents Chemother. 42, 2590-2594.

Bhateja, P., Purnapatre, K., Dube, S., Fatma, T., and Rattan, A. (2006). Characterisation of laboratory-generated vancomycin intermediate resistant Staphylococcus aureus strains. Int. J. Antimicrob. Agents 27, 201-211. doi: 10.1016/j.ijantimicag.2005.10.008

Cameron, D. R., Jiang, J. H., Kostoulias, X., Foxwell, D. J., and Peleg, A. Y. (2016). Vancomycin susceptibility in methicillin-resistant Staphylococcus aureus is mediated by YycHI activation of the WalRK essential two-component regulatory system. Sci. Rep. 6:30823. doi: 10.1038/srep30823

Chang, S., Sievert, D. M., Hageman, J. C., Boulton, M. L., Tenover, F. C., Downes, F. P., et al. (2003). Infection with vancomycin-resistant Staphylococcus aureus containing the vanA resistance gene. N.Engl. J. Med. 348, 1342-1347. doi: 10.1056/NEJMoa025025

Cockerill, F. R. (2012). Methods for Dilution Antimicrobial Susceptibility Tests for Bacteria That Grow Aerobically: Approved Standard. Wayne, PA: Clinical and Laboratory Standards Institute.

Cui, L., Neoh, H. M., Shoji, M., and Hiramatsu, K. (2009). Contribution of vraSR and graSR point mutations to vancomycin resistance in vancomycinintermediate Staphylococcus aureus. Antimicrob. Agents Chemother. 53, 1231-1234. doi: 10.1128/AAC.01173-08

Dantes, R., Mu, Y., Belflower, R., Aragon, D., Dumyati, G., Harrison, L. H., et al. (2013). National burden of invasive methicillin-resistant Staphylococcus aureus infections, United States, 2011. JAMA Intern. Med. 173, 1970-1978. doi: 10.1001/jamainternmed.2013.10423

Deresinski, S. (2005). Methicillin-resistant Staphylococcus aureus: an evolutionary, epidemiologic, and therapeutic odyssey. Clin. Infect. Dis. 40, 562-573. doi: $10.1086 / 427701$

Doddangoudar, V. C., O’Donoghue, M. M., Chong, E. Y., Tsang, D. N., and Boost, M. V. (2012). Role of stop codons in development and loss of vancomycin non-susceptibility in methicillin-resistant Staphylococcus aureus. J. Antimicrob. Chemother. 67, 2101-2106. doi: 10.1093/jac/dks171

Drlica, K. (2003). The mutant selection window and antimicrobial resistance. J. Antimicrob. Chemother. 52, 11-17. doi: 10.1093/jac/dkg269

Enright, M. C., Day, N. P., Davies, C. E., Peacock, S. J., and Spratt, B. G. (2000). Multilocus sequence typing for characterization of methicillin-resistant and methicillin-susceptible clones of Staphylococcus aureus. J. Clin. Microbiol. 38, 1008-1015.

Gao, W., Cameron, D. R., Davies, J. K., Kostoulias, X., Stepnell, J., Tuck, K. L., et al. (2013). The RpoB H148Y rifampicin resistance mutation and an active stringent response reduce virulence and increase resistance to innate immune responses in Staphylococcus aureus. J. Infect. Dis. 207, 929-939. doi: 10.1093/infdis/jis772

Gardete, S., Kim, C., Hartmann, B. M., Mwangi, M., Roux, C. M., Dunman, P. M., et al. (2012). Genetic pathway in acquisition and loss of vancomycin resistance in a methicillin resistant Staphylococcus aureus (MRSA) strain of clonal type USA300. PLoS Pathog. 8:e1002505. doi: 10.1371/journal.ppat. 1002505

Hafer, C., Lin, Y., Kornblum, J., Lowy, F. D., and Uhlemann, A. C. (2012). Contribution of selected gene mutations to resistance in clinical isolates of vancomycin-intermediate Staphylococcus aureus. Antimicrob. Agents Chemother. 56, 5845-5851. doi: 10.1128/AAC.01139-12

Hidron, A. I., Edwards, J. R., Patel, J., Horan, T. C., Sievert, D. M., Pollock, D. A., et al., (2008). NHSN annual update: antimicrobial-resistant pathogens associated with healthcare-associated infections: annual summary of data reported to the National Healthcare Safety Network at the Centers for Disease Control and Prevention, 2006-2007. Infect. Control Hosp. Epidemiol. 29, 996-1011. doi: 10.1086/591861
Hiramatsu, K., Aritaka, N., Hanaki, H., Kawasaki, S., Hosoda, Y., Hori, S., et al. (1997a). Dissemination in Japanese hospitals of strains of Staphylococcus aureus heterogeneously resistant to vancomycin. Lancet 350, 1670-1673.

Hiramatsu, K., Hanaki, H., Ino, T., Yabuta, K., Oguri, T., and Tenover, F. C. (1997b). Methicillin-resistant Staphylococcus aureus clinical strain with reduced vancomycin susceptibility. J. Antimicrob. Chemother. 40, 135-136.

Hiramatsu, K., Okuma, K., Ma, X. X., Yamamoto, M., Hori, S., and Kapi, M. (2002). New trends in Staphylococcus aureus infections: glycopeptide resistance in hospital and methicillin resistance in the community. Curr. Opin. Infect. Dis. 15, 407-413. doi: 10.1097/00001432-200208000-00009

Howden, B. P., McEvoy, C. R., Allen, D. L., Chua, K., Gao, W., Harrison, P. F., et al. (2011). Evolution of multidrug resistance during Staphylococcus aureus infection involves mutation of the essential two component regulator WalKR. PLoS Pathog. 7:e1002359. doi: 10.1371/journal.ppat.1002359

Howden, B. P., Smith, D. J., Mansell, A., Johnson, P. D., Ward, P. B., Stinear, T. P., et al. (2008a). Different bacterial gene expression patterns and attenuated host immune responses are associated with the evolution of low-level vancomycin resistance during persistent methicillin-resistant Staphylococcus aureus bacteraemia. BMC Microbiol. 8:39. doi: 10.1371/journal.ppat.1002359

Howden, B. P., Stinear, T. P., Allen, D. L., Johnson, P. D., Ward, P. B., and Davies, J. K. (2008b). Genomic analysis reveals a point mutation in the two-component sensor gene graS that leads to intermediate vancomycin resistance in clinical Staphylococcus aureus. Antimicrob. Agents Chemother. 52, 3755-3762. doi: 10.1128/AAC.01613-07

Jonckheere, A. R. (1954). A distribution-free k-sample test against ordered alternatives. Biometrika 41, 133-145. doi: 10.1093/biomet/41.1-2.133

Kallen, A. J., Mu, Y., Bulens, S., Reingold, A., Petit, S., Gershman, K., et al., (2010). Health care-associated invasive MRSA infections, 2005-2008. J. Am. Med. Assoc. 304, 641-648. doi: 10.1001/jama.2010.1115

Katayama, Y., Murakami-Kuroda, H., Cui, L., and Hiramatsu, K. (2009). Selection of heterogeneous vancomycin-intermediate Staphylococcus aureus by imipenem. Antimicrob. Agents Chemother. 53, 3190-3196. doi: 10.1128/AAC.00834-08

Kato, Y., Suzuki, T., Ida, T., and Maebashi, K. (2010). Genetic changes associated with glycopeptide resistance in Staphylococcus aureus: predominance of amino acid substitutions in YvqF/VraSR. J. Antimicrob. Chemother. 65, 37-45. doi: $10.1093 / \mathrm{jac} / \mathrm{dkp} 394$

Klevens, R. M., Morrison, M. A., Nadle, J., Petit, S., Gershman, K., Ray, S., et al. (2007). Invasive methicillin-resistant Staphylococcus aureus infections in the United States. J. Am. Med. Assoc. 298, 1763-1771. doi: 10.1001/jama.298.15.1763

Liu, C., Bayer, A., Cosgrove, S. E., Daum, R. S., Fridkin, S. K., Gorwitz, R. J., et al. (2011). Clinical practice guidelines by the infectious diseases society of america for the treatment of methicillin-resistant Staphylococcus aureus infections in adults and children: executive summary. Clin. Infect. Dis. 52, 285-292. doi: 10.1093/cid/cir034

Matsuo, M., Hishinuma, T., Katayama, Y., Cui, L., Kapi, M., and Hiramatsu, K. (2011). Mutation of RNA polymerase beta subunit ( $r p o B)$ promotes hVISA-toVISA phenotypic conversion of strain Mu3. Antimicrob. Agents Chemother. 55, 4188-4195. doi: 10.1128/AAC.00398-11

Mick, V., Domínguez, M. A., Tubau, F., Liñares, J., Pujol, M., and Martín, R. (2010). Molecular characterization of resistance to Rifampicin in an emerging hospital-associated Methicillin-resistant Staphylococcus aureus clone ST228, Spain. BMC Microbiol. 10:68. doi: 10.1186/1471-2180-10-68

Moellering, R. C. Jr. (2005). The management of infections due to drugresistant gram-positive bacteria. Eur. J. Clin. Microbiol. Infect. Dis. 24, 777-779. doi: 10.1007/s10096-005-0062-2

Mwangi, M. M., Wu, S. W., Zhou, Y., Sieradzki, K., de Lencastre, H., Richardson, P., et al. (2007). Tracking the in vivo evolution of multidrug resistance in Staphylococcus aureus by whole-genome sequencing. Proc. Natl. Acad. Sci. U.S.A. 104, 9451-9456. doi: 10.1073/pnas.0609839104

Neoh, H. M., Cui, L., Yuzawa, H., Takeuchi, F., Matsuo, M., and Hiramatsu, K. (2008). Mutated response regulator graR is responsible for phenotypic conversion of Staphylococcus aureus from heterogeneous vancomycinintermediate resistance to vancomycin-intermediate resistance. Antimicrob. Agents Chemother. 52, 45-53. doi: 10.1128/AAC.00534-07

O'Neill, A. J., Huovinen, T., Fishwick, C. W., and Chopra, I. (2006). Molecular genetic and structural modeling studies of Staphylococcus aureus 
RNA polymerase and the fitness of rifampin resistance genotypes in relation to clinical prevalence. Antimicrob. Agents Chemother. 50, 298-309. doi: 10.1128/AAC.50.1.298-309.2006

Patel, J. B. (2014). Performance Standards for Antimicrobial Susceptibility Testing: Twenty-Fourth Informational Supplement. Wayne, PA: Clinical and Laboratory Standards Institute.

Popovich, K., Hota, B., Rice, T., Aroutcheva, A., and Weinstein, R. A. (2007). Phenotypic prediction rule for community-associated methicillinresistant Staphylococcus aureus. J. Clin. Microbiol. 45, 2293-2295. doi: 10.1128/JCM.00044-07

Rishishwar, L., Kraft, C. S., and Jordan, I. K. (2016). Population genomics of reduced vancomycin susceptibility in Staphylococcus aureus. mSphere 1:e0009416. doi: $10.1128 / \mathrm{mSphere.00094-16}$

Sader, H. S., Jones, R. N., Rossi, K. L., and Rybak, M. J. (2009). Occurrence of vancomycin-tolerant and heterogeneous vancomycin-intermediate strains (hVISA) among Staphylococcus aureus causing bloodstream infections in nine USA hospitals. J. Antimicrob. Chemother. 64, 1024-1028. doi: $10.1093 /$ jac/dkp319

Saha, B., Singh, A. K., Ghosh, A., Bal, M. (2008). Identification and characterization of a vancomycin-resistant Staphylococcus aureus isolated from Kolkata (South Asia). J. Med. Microbiol. 57, 72-79. doi: 10.1099/jmm.0.47144-0

Sakoulas, G., Eliopoulos, G. M., Fowler, V. G., Moellering, R. C., Novick, R. P., Lucindo, N., et al. (2005). Reduced susceptibility of Staphylococcus aureus to vancomycin and platelet microbicidal protein correlates with defective autolysis and loss of accessory gene regulator (agr) function. Antimicrob. Agents Chemother. 49, 2687-2692. doi: 10.1128/AAC.49.7.2687-2692. 2005

Sakoulas, G., Eliopoulos, G. M., Moellering, R. C., Wennersten, C., Venkataraman, L., Novick, R. P., et al. (2002). Accessory gene regulator (agr) locus in geographically diverse Staphylococcus aureus isolates with reduced susceptibility to vancomycin. Antimicrob. Agents Chemother. 46, 1492-1502. doi: 10.1128/AAC.46.5.1492-1502. 2002
Shoji, M., Cui, L., Iizuka, R., Komoto, A., Neoh, H. M., Watanabe, Y., et al. (2011). walK and clpP mutations confer reduced vancomycin susceptibility in Staphylococcus aureus. Antimicrob. Agents Chemother. 55, 3870-3881. doi: 10.1128/AAC.01563-10

Sieradzki, K., Roberts, R. B., Haber, S. W., and Tomasz, A. (1999). The development of vancomycin resistance in a patient with methicillinresistant Staphylococcus aureus infection. N. Engl. J. Med. 340, 517-523. doi: 10.1056/NEJM199902183400704

Tenover, F. C., and Moellering, R. C., Jr. (2007). The rationale for revising the clinical and laboratory standards institute vancomycin minimal inhibitory concentration interpretive criteria for Staphylococcus aureus. Clin. Infect. Dis. 44, 1208-1215. doi: 10.1086/513203

Tibshirani, R., Walther, G., and Hastie, T. (2001). Estimating the number of clusters in a data set via the gap statistic. J. R. Statist. Soc. B 63, 411-423. doi: 10.1111/1467-9868.00293

Watanabe, Y., Cui, L., Katayama, Y., Kozue, K., and Hiramatsu, K. (2011). Impact of rpoB mutations on reduced vancomycin susceptibility in Staphylococcus aureus. J. Clin. Microbiol. 49, 2680-2684. doi: 10.1128/JCM.02144-10

Conflict of Interest Statement: The authors declare that the research was conducted in the absence of any commercial or financial relationships that could be construed as a potential conflict of interest.

The reviewer SA and handling Editor declared their shared affiliation and the handling Editor states that the process nevertheless met the standards of a fair and objective review.

Copyright (C) 2017 Wang, Li, Jiang, Han, Xie, Jin, He and Wu. This is an open-access article distributed under the terms of the Creative Commons Attribution License (CC $B Y)$. The use, distribution or reproduction in other forums is permitted, provided the original author(s) or licensor are credited and that the original publication in this journal is cited, in accordance with accepted academic practice. No use, distribution or reproduction is permitted which does not comply with these terms. 